\title{
Understanding long-range near-side ridge correlations in $p$ - $p$ collisions using rope hadronization at energies available at the CERN Large Hadron Collider
}

\author{
Pritam Chakraborty* and Sadhana Dash ${ }^{\dagger}$ \\ Indian Institute of Technology Bombay, Mumbai, 400076, India
}

(Received 30 June 2020; accepted 24 September 2020; published 11 November 2020)

\begin{abstract}
The observation of long range ridge-like structure in the near-side region of the two particle $\Delta \eta-\Delta \phi$ correlations as measured by CERN Large Hadron Collider experiments in high multiplicity $p$ - $p$ collisions indicated towards the presence of collective effects which are similar to that observed in $p$ - $A$ (nucleon-nucleus) and $A-A$ (nucleus-nucleus) collisions. The two particle correlation between the charged particles in $\Delta \eta-\Delta \phi$ for $p$ - $p$ collisions at $\sqrt{s}=7 \mathrm{TeV}$ and $13 \mathrm{TeV}$ is studied using the PYTHIA 8 event generator within the framework of final-state partonic color reconnection effects as well as the microscopic rope hadronization model. The rope hadronization relies on the formation of ropes due to overlapping of strings in high multiplicity events followed by string shoving. A near side ridge-like structure which is qualitatively similar to the observed ridge in data was observed for high-multiplicity events when the mechanism of rope hadronization (with shoving) was enabled.
\end{abstract}

DOI: 10.1103/PhysRevC.102.055202

\section{INTRODUCTION}

The recent observation of long-range ridge like structure in the near side region of $\Delta \eta-\Delta \phi$ correlations of charged particle pairs measured by the CMS and ATLAS experiment at the CERN Large Hadron Collider (LHC) [1-3] has generated a lot of interest in small systems. An enhanced correlation was found for charged particle pairs which are highly collimated in azimuthal angle over large $\eta$ intervals. These ridge-like structures and long range correlations were not previously observed in minimum bias events of hadronic collisions. Such observations are reminiscent of ridge-like features present in heavy ion collisions and are believed to be manifestations of hydrodynamic collectivity in partonic matter [4,5]. However, it is also expected that no-QGP like effects should be seen in $p$ - $p$ collisions as the size of system formed in hadronic collisions is extremely small and short lived. As the particle production in high multiplicity events in hadronic collisions are highly influenced by nonperturbative QCD processes, the study of such events can provide information about the potential microscopic processes leading to such novel observations.

The CMS experiment at LHC observed a nontrivial ridgelike structure for the first time in the near side, long range region of the two-dimensional $\Delta \eta-\Delta \phi$ distributions of

\footnotetext{
*pritam@iitb.ac.in

†sadhana@phy.iitb.ac.in
}

Published by the American Physical Society under the terms of the Creative Commons Attribution 4.0 International license. Further distribution of this work must maintain attribution to the author(s) and the published article's title, journal citation, and DOI. Funded by SCOAP . charged particle pairs in high multiplicity $p-p$ collisions at $\sqrt{s}=7 \mathrm{TeV}$ and $13 \mathrm{TeV}[1,2]$. The correlation structure was found to be more pronounced in the intermediate $p_{T}$ range and for high multiplicity events. It is well known that the two particle correlation function in minimum bias events is conspicuous with its enhanced structure due to contributions from jet fragmentation, resonance decays, string fragmentation, etc., at $(\Delta \eta, \Delta \phi) \sim(0,0)$. The fragmentation of back-to-back jets contribute a broad ridge-like structure around $\Delta \phi \approx \pi$ and the principle of momentum conservation leads to a shallow dip at $\Delta \phi=0$ for larger $\Delta \eta$ ranges. However, in nucleus-nucleus $(A-A)$ collisions, a ridge-like structure was also observed in the near-side region around $\Delta \phi=0$ for large values of $\Delta \eta$ for correlated pairs of hadrons as measured by BNL Relativistic Heavy Ion Collider (RHIC) and LHC experiments $[4,5]$. The strength (or amplitude) of these correlations increased with collision centrality. This observation of near-side long range rapidity correlation was attributed to the presence of strong collectivity of the matter created in heavy ion collisions [6]. However, many theories related to the response of the medium to the partonic interactions, initial fluctuations in the geometry of overlapping nuclei leading to triangularity (and higher order) final state azimuthal anisotropy, etc., were also investigated to understand the novel phenomenon [7-9].

Previously, long range correlations were also observed in hadronic collisions at ISR experiments but they were not collimated in azimuthal angle and thus did not resemble a ridge [10]. The LHC experiments also studied the two particle correlations in high multiplicity $p$ - $\mathrm{Pb}$ collisions and observed the striking ridge-like correlations in the near side, long range region [11]. However, the magnitude of the structure was found to be five times higher than that observed in $p$ - $p$ collisions. Many alternative explanations based on hydrodynamic evolution [12] and the formation of color glass 
condensate $[13,14]$ could explain the observation of ridge in $p$ - $p$ collisions. A study carried out in the context of correlated cluster model where the emission of correlated clusters in the azimuthal space was considered also provided a common framework to explain the ridge structure in different collision systems $[15,16]$. The mechanism of string percolation outlines in $[17,18]$ also provides a complementary picture to understand the near-side ridge correlations. A recent study using the DIPSY generator [20] which incorporated the shoving mechanism in rope hadronization could qualitatively explain the collective phenomenon of the formation of near side ridge in long range correlations for $p$ - $p$ collisions [21]. However, the study was shown in a biased sample of DIPSY events where the events had only long strings. In this work, the two particle correlation of charged particles in $\Delta \eta-\Delta \phi$ is investigated in details using the shoving mechanism implemented in PYTHIA 8 [19] event generator for $p$ - $p$ collisions at $\sqrt{s}=7 \mathrm{TeV}$ and $13 \mathrm{TeV}$. The theory of color reconnections and string hadronization via the formation of ropes could explain the strangeness enhancement and the suppression of yields of resonance particles in $p$ - $p$ collisions at $\sqrt{s}=7 \mathrm{TeV}$ and 13 TeV using PYTHIA 8 [22-25]. The model does not assume the formation of a deconfined and thermalized plasma state due to the dynamical interactions between the partons. In $p$ - $p$ collisions at high energy, one can have multitude of partonic interactions in a single event which is more pronounced in high multiplicity events. The various subprocesses during multipartonic interactions (MPI) are reconnected and the color flow is reassigned in the beam remnant by different processes which define the different mode of color reconnection mechanism. The mechanism of color reconnections refers to color connections between the final partonic strings before hadronization. The MPI-mode (CR-0) refers to the fusion of color flow so that to yield the smallest string length. For CR-0 scheme, the partons from lower $p_{T}$ in the considered MPI systems are added to the dipoles defined by the higher $p_{T}$ system such that that the total string length is minimized. The QCD mode (CR-1) incorporates a complete treatment of the QCD multiplets (based on color rules) and can also produce three color indices structure called junctions. This leads to an enhancement of baryons. In the gluon-move scheme (CR-2), only gluons are considered for reconnections to all the MPI systems irrespective of the $p_{T}$ and hence the color flow from hard interactions get affected compared to the CR-0 scheme. The reconnections for all schemes are based on minimization of the string length [26,27]. In high multiplicity events, these color strings overlap with each other and act coherently to form a color rope that subsequently hadronize with a higher effective string tension [28,29]. The greater energy density of the string overlap region creates a dynamic pressure gradient which push the strings in the outward direction. The $p_{T}$, transverse momentum, of the strings is generated from the excess energy in the central region. Due to this outward movement of strings, the pressure gradient as well as the excess energy gradually decreases and consequently the strings pick less $p_{T}$ until there is no overlap. Thus, these strings shove each other picking up extra transverse boost and hence an increase of mean $p_{T}$ for heavier hadrons is observed. This observation is similar to the that obtained in case of hydrodynamic scenario.
The effects of different modes of color reconnections are also studied for both the energies.

\section{ANALYSIS METHOD}

Generally, the two particle correlations are studied in terms of two-dimensional $\Delta \eta-\Delta \phi$ correlation functions [1,2]. In this work, the generated events are classified into different class of multiplicities depending on the number of particles produced within $|\eta|<2.4$ and $p_{T}>0.4 \mathrm{GeV} / c$. For a given multiplicity class, the trigger particles are selected from a given $p_{T}$ range and the total number of trigger particles is referred to as $N_{\text {trig. }}$. Each trigger particle is then associated with the remaining charged particles (called associated particles) from a certain $p_{T}$ range to form particle pairs. The per trigger particle yield of such pairs from same event is $S(\Delta \eta, \Delta \phi)$ and is expressed as

$$
S(\Delta \eta, \Delta \phi)=\frac{1}{N_{\text {trig }}} \frac{d^{2} N^{\text {same }}}{d \Delta \eta \Delta \phi} .
$$

The symbols $\Delta \eta$ and $\Delta \phi$ denote the differences in $\eta$ and $\phi$ of the formed pair. The mixed event pair distribution is constructed by forming pairs of the trigger particles in a given event with the associated particles from different events. The number of events mixed for this analysis was 10 . The mixedevent pair distribution is given by

$$
B(\Delta \eta, \Delta \phi)=\frac{1}{N_{\text {trig }}} \frac{d^{2} N^{\text {mix }}}{d \Delta \eta \Delta \phi} .
$$

The distribution of signal pairs and background pairs are constructed event wise for a given multiplicity class and are then averaged over all considered events to get the distribution of the corrected per trigger particle associated yield. The corrected per trigger particle associated yield is then defined as

$$
\frac{1}{N_{\text {trig }}} \frac{d^{2} N^{\text {pair }}}{d \Delta \eta d \Delta \phi}=B(0,0) \frac{S(\Delta \eta, \Delta \phi)}{B(\Delta \eta, \Delta \phi)} .
$$

The PYTHIA 8 event generator [19] was used to generate 100 and 80 million events for $p$ - $p$ collisions at $\sqrt{s}=7 \mathrm{TeV}$ and $13 \mathrm{TeV}$, respectively. The events were analysed to obtain the two dimensional $\Delta \eta-\Delta \phi$ correlation functions for charged particles in different multiplicity classes. The trigger and associated particles have been chosen from the $p_{T}$ ranges, $0.5-3 \mathrm{GeV} / c$ and $1-3 \mathrm{GeV} / c$ for three different multiplicity class ranging from $0<N_{\text {trk }}<20,80<N_{\text {trk }}<100$, and $N_{\text {trk }}>100$. The $N_{\text {trk }}$ refers to the number of charged particles within the acceptance of $|\eta|<0.5$. The Monash 2013 tune of PYTHIA 8.2 [30] has been used to generate events for $p$ - $p$ collisions. The multipartonic interactions were enabled for the study. The three different modes of color reconnection mechanism [namely MPI-based(CR-0), QCD -based(CR-1), and gluon-move(CR-2)] were studied with (and without) the rope hadronization scenario to observe the effect of color reconnection and rope hadronization on two particle correlations. For the CR0 scheme, the partons from lower $p_{T}$ in the considered MPI systems are added to the dipoles defined by the higher $p_{T}$ system such that that the total string length is minimized. 


\section{RESULTS}

The two-dimensional $\Delta \eta-\Delta \phi$ correlation functions for charged particle pairs (charged trigger particle and charged associated particle) are shown for two different multiplicity classes in $p$ - $p$ collisions at $\sqrt{s}=7 \mathrm{TeV}$ and $13 \mathrm{TeV}$ in Figs. 1 and 2, respectively. The figures also compare the

$\mathrm{CR}=2, \mathrm{RH}=$ off, $0<\mathrm{N}<20$

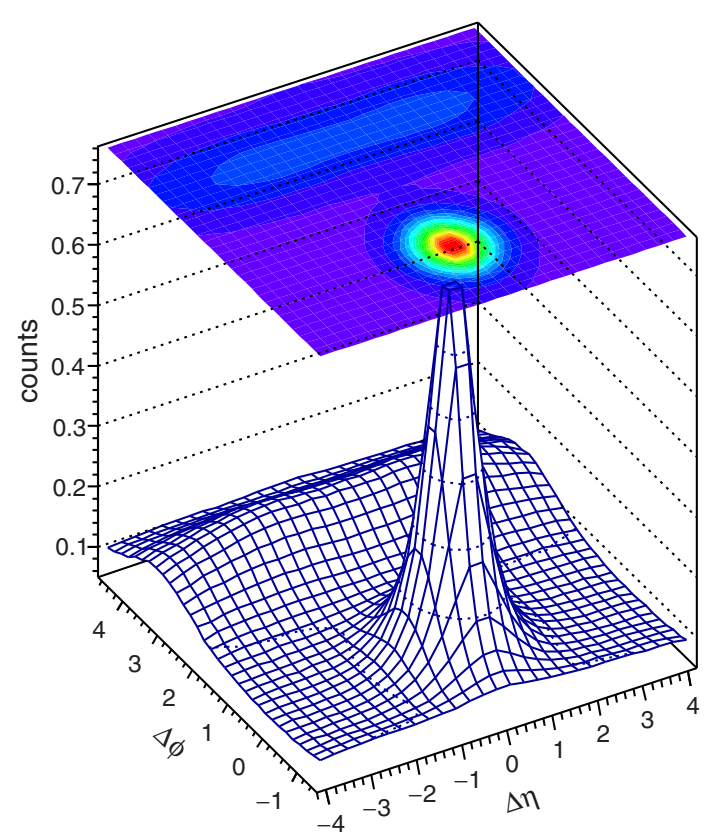

$\mathrm{CR}=2, \mathrm{RH}=\mathrm{on}, 0<\mathrm{N}<20$

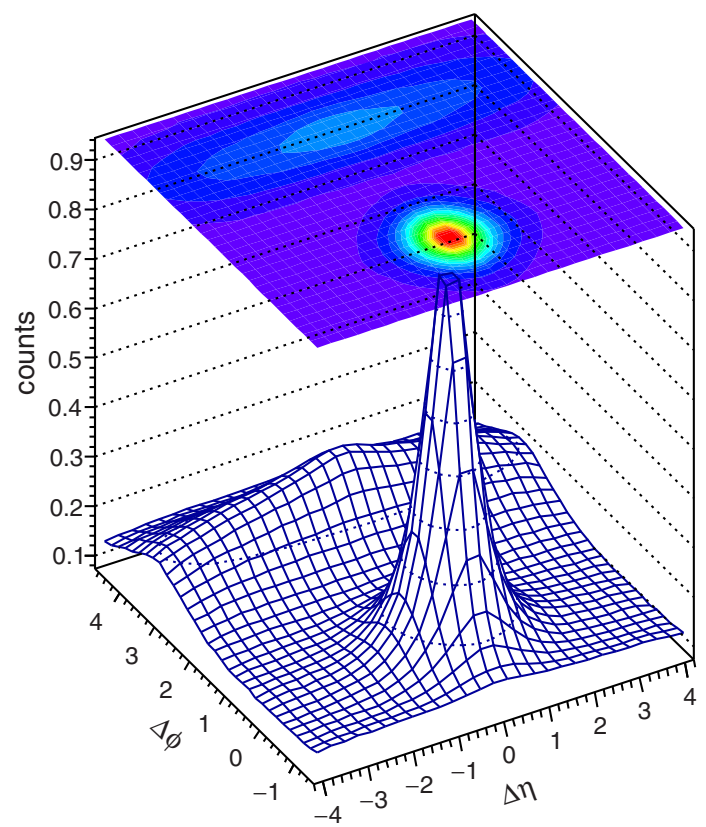

correlation function of the charged particles for the effect of rope hadronization in presence of color reconnections for the considered multiplicity classes. An expected correlation peak near $\Delta \eta-\Delta \phi=(0,0)$ is observed for both low and high multiplicity class for both the energies. The peak primarily originates due to jet fragmentation as mentioned before.
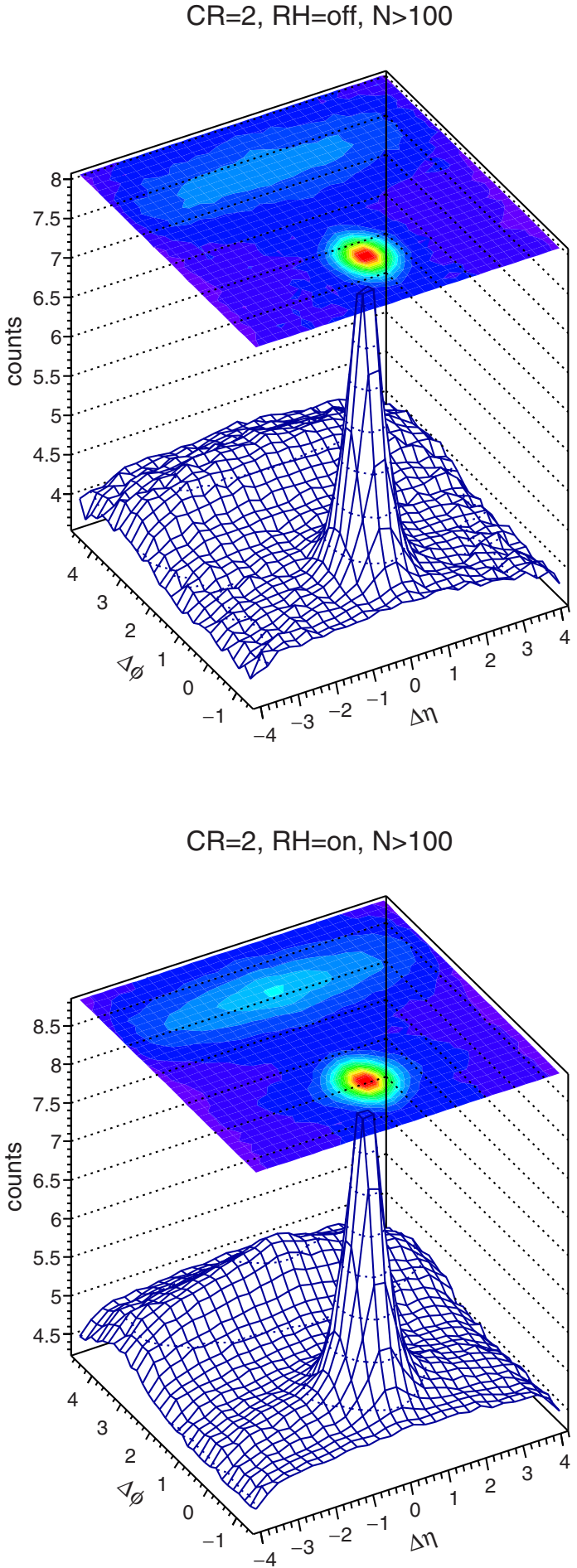

FIG. 1. The two-dimensional $\Delta \eta-\Delta \phi$ correlation function of charged particle pairs in $p$ - $p$ collisions at $\sqrt{s}=7$ TeV for two different multiplicity classes with $1.0<p_{T}^{\text {Trigg }}<3.0 \mathrm{GeV} / c$ and $1<p_{T}($ Asso $)<3.0$. The top panels refer to the case when rope formation is not considered while the bottom panels show the same in the presence of rope hadronization. 
$\mathrm{CR}=2, \mathrm{RH}=\mathrm{off}, 0<\mathrm{N}<20$

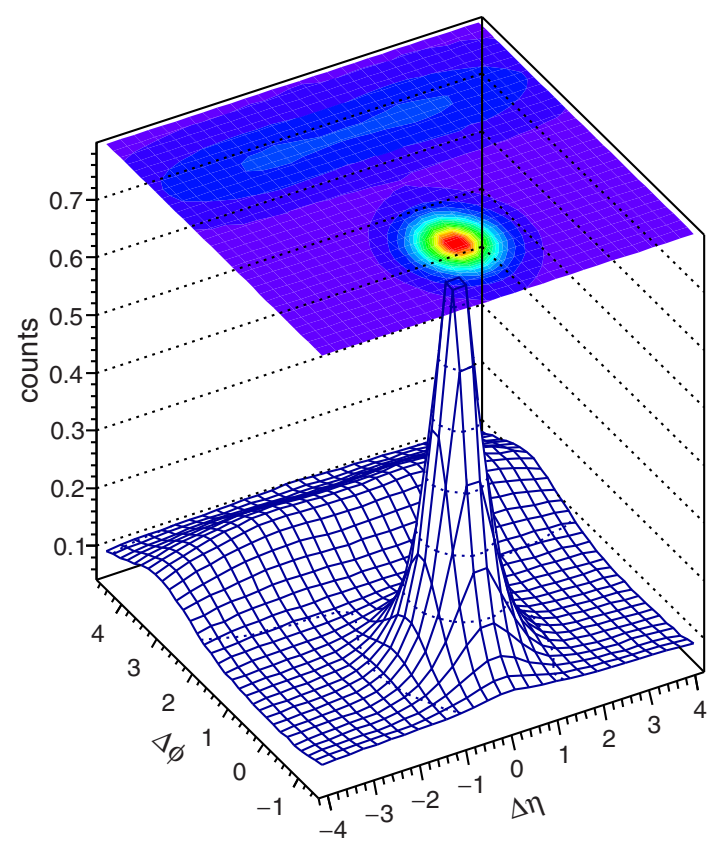

$\mathrm{CR}=2, \mathrm{RH}=\mathrm{on}, 0<\mathrm{N}<20$

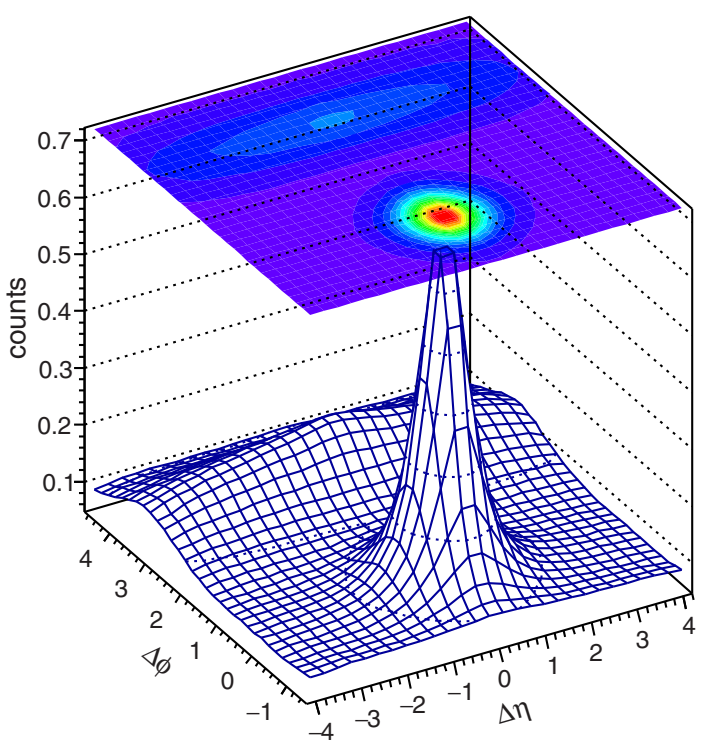

$\mathrm{CR}=2, \mathrm{RH}=\mathrm{off}, \mathrm{N}>100$

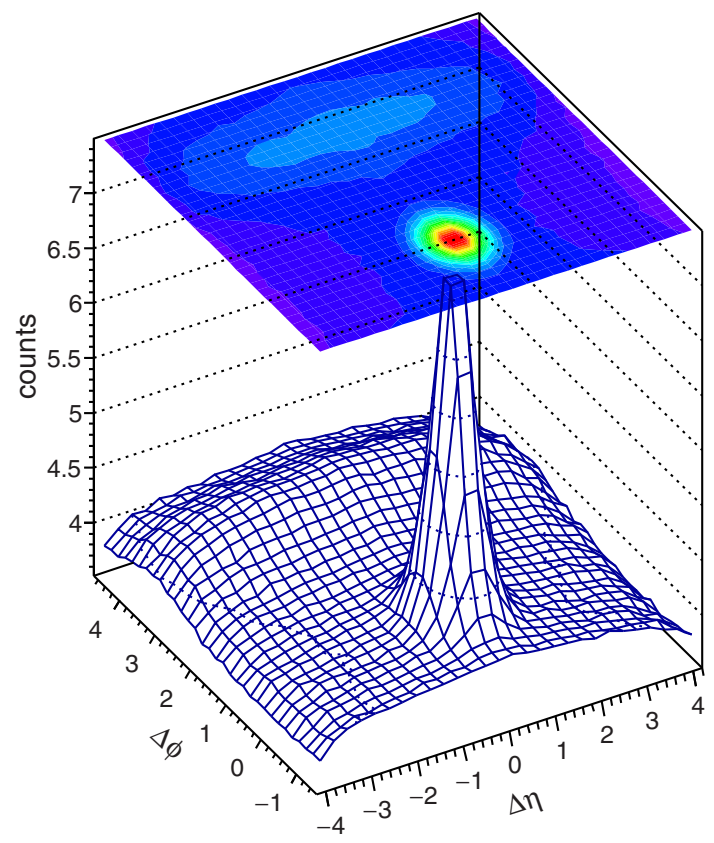

$\mathrm{CR}=2, \mathrm{RH}=\mathrm{on}, \mathrm{N}>100$

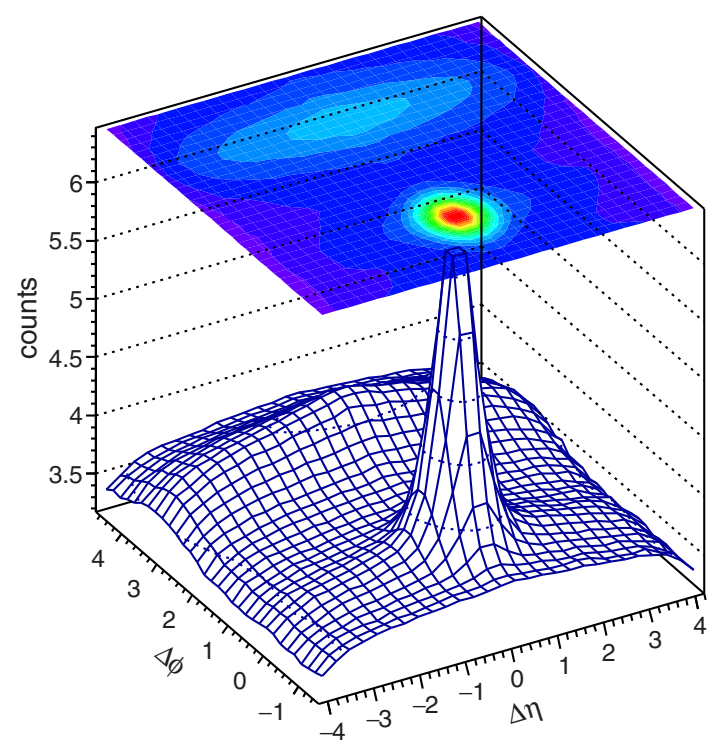

FIG. 2. The two-dimensional $\Delta \eta-\Delta \phi$ correlation function of charged particle pairs in $p$ - $p$ collisions at $\sqrt{s}=13$ TeV for two different multiplicity classes with $1.0<p_{T}^{\text {Trigg }}<3.0 \mathrm{GeV} / c$ and $1<p_{T}$ (Asso) $<3.0$. The top panels refer to the case when rope formation is not considered while the bottom panels show the same in the presence of rope hadronization.

An away side $(\Delta \phi \approx \pi)$ ridge-like structure extended up to higher values of $|\Delta \eta|$ (containing the contributions from back-to-back jets) is also visible in all the figures. However, a long-range ridge-like structure extending almost three units in $\Delta \eta$ can be seen in the near side $(\Delta \phi \approx 0)$ region of the correlation function for high multiplicity class where the formation of ropes is more pronounced. This structure is not observed in low multiplicity class where the formation of ropes is less probable due to lack of overlapping strings. Moreover, the structure is not present in higher multiplicity class when the formation of ropes in not considered. This ridge-like structure is quite similar to the observed ridge in high multiplicity $p$ - $p$ collisions. The details of the observed two-dimensional correlation functions were further investigated by projecting the event-averaged distributions into one-dimensional distribution in $\Delta \phi$ for different $\Delta \eta$ ranges. The projections over different $\Delta \eta$ range were divided into two groups, namely the short range (when projected over $|\Delta \eta|<1.0$, the jet region) 

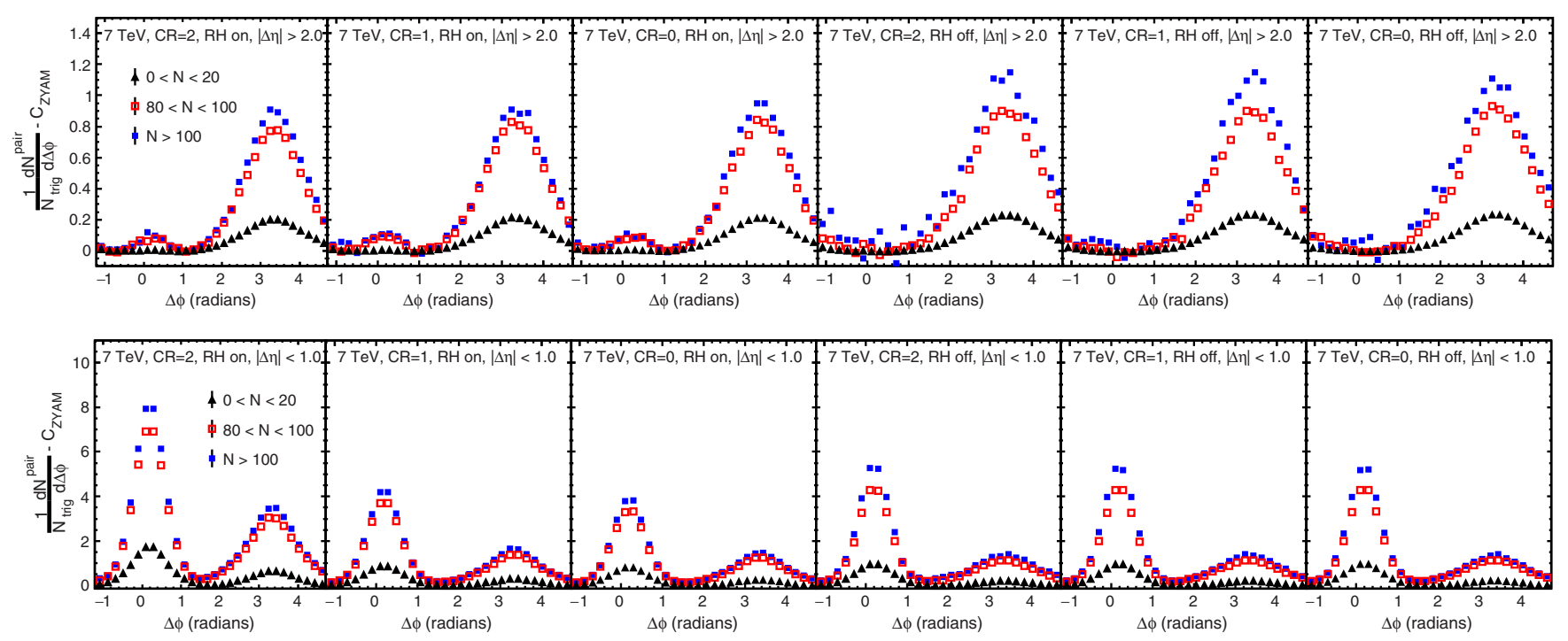

FIG. 3. The one-dimensional $\Delta \phi$ projection of two particle correlation function of charged particles in $p$ - $p$ collisions at $\sqrt{s}=7$ TeV for three different multiplicity classes. The top panels refer to the long range region $(\Delta \eta>2.0)$ while the lower ones refer to the short range region $(\Delta \eta<1.0)$.

and the long range $(|\Delta \eta|>2.0$, the ridge region). The standard zero-yield-at-minimum (ZYAM) method is employed to estimate the associated yield in the correlated region [31]. In this method, the one-dimensional $\Delta \phi$ correlation distribution is fitted with a second order polynomial function in the range of $0.1<|\Delta \phi|<2$. The lowest value of the fitted polynomial function, $C_{\mathrm{ZYAM}}$ (which accounts for the constant pedestal) is subtracted from the distribution. As a result, the minimum of the correlation function has zero associated yield. This procedure is implemented to correct the one-dimensional $\Delta \phi$ correlation function in $p$ - $p$ collisions at both the energies for both long- and short-range region. This study was performed for three different multiplicity classes with three different modes of color reconnections within (and without) the framework of rope hadronization. Figures 3 and 4 compare the effect of rope hadronization in one-dimensional $\Delta \phi$ correlations for three different modes of color reconnections in $p$ - $p$ collisions at 7 and $13 \mathrm{TeV}$, respectively. The top panels which focus on long range region show an expected awayside peak emanating from back-to-back jets for all the three multiplicity classes. However, a nonzero associated yield peak is also observed in the near-side for long-range region for high multiplicity events when the rope hadronization is enabled. This peak is absent for low multiplicity events as well as for events without rope hadronization for both the energies. This observation indicates that the microscopic phenomenon
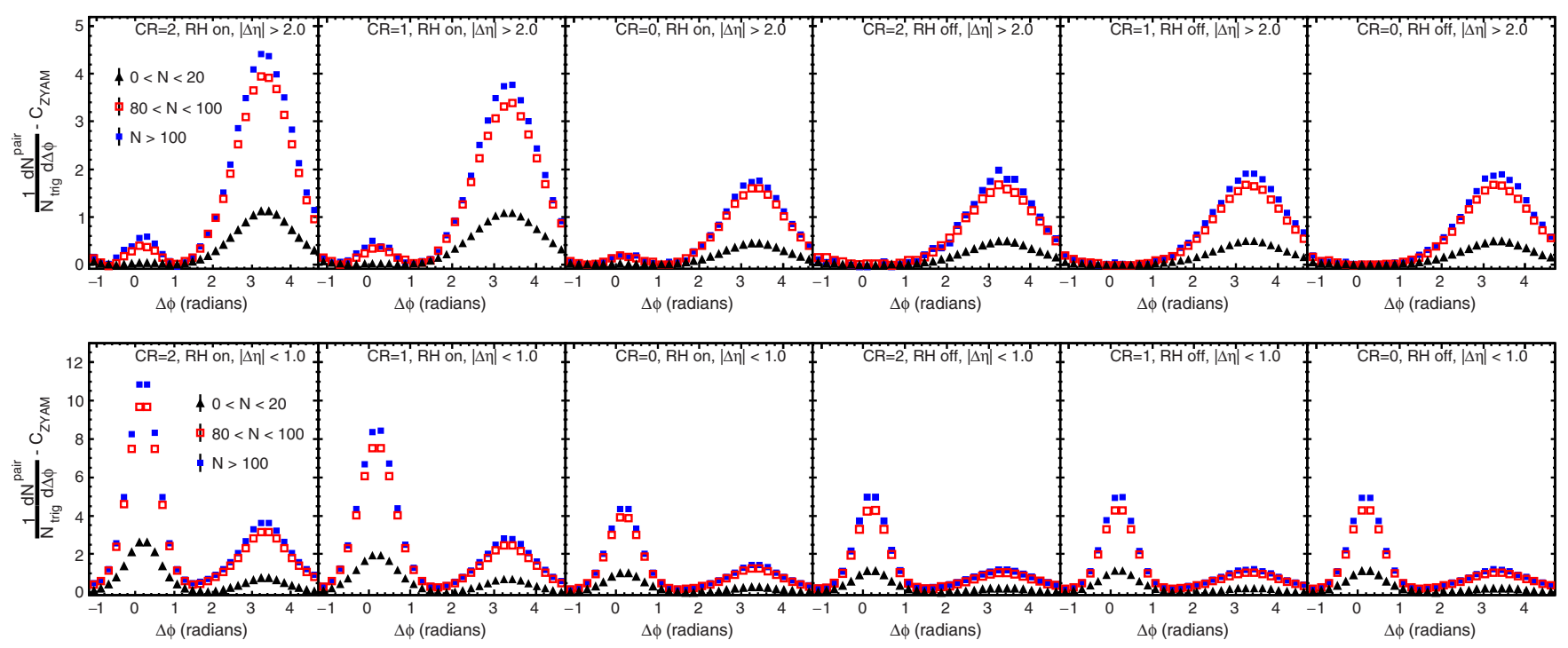

FIG. 4. The one-dimensional $\Delta \phi$ projection of two particle correlation function of charged particles in $p$ - $p$ collisions at $\sqrt{s}=13$ TeV for three different multiplicity classes. The top panels refer to the long range region $(\Delta \eta>2.0)$ while the lower ones refer to the short range region $(\Delta \eta<1.0)$. 
of partonic color reconnections together with the formation of ropes due to overlapping strings followed by shoving and subsequent hadronization in high multiplicity events can also generate ridge-like structure in the near side region. The bottom panels of the figure show the short range region and one can observe the expected near side peak originating due to jets and an away-side ridge-like structure (due to back-to back jets and momentum conservation).The strength of the correlation function is higher for the CR-1 and CR-2 modes compared to the CR-0 mode. This may be attributed to additional reconnections in the former modes due to junction formation and shifting the gluon position in order to minimize the string length. A clear beam energy dependence is also observed with respect to the magnitude of peaks in both the near-side and away-side region. A detailed study with identified particles is required to gain more understanding. Nevertheless, the observed ridge-like structure in the near-side region is qualitatively similar to the ones observed in data which further supports the idea that the microscopic processes at partonic level can mimic collectivity like features without assuming the formation of a thermalized medium.

\section{SUMMARY}

The observation of long range $\Delta \eta-\Delta \phi$ correlations of charged particles at near-side $(\Delta \phi \approx 0)$ measured by LHC experiments in high multiplicity $p$ - $p$ collisions indicated towards the presence of small scale collective effects which are similar to that observed in $p-A$ (nucleon-nucleus) and $A-A$ (nucleus-nucleus) collisions. This novel observation was investigated by studying the two particle correlation between charged particles in $p$ - $p$ collisions at $\sqrt{s}=7 \mathrm{TeV}$ and 13 TeV using PYTHIA 8 event generator within the framework of final-state partonic color reconnection mechanism as well as the microscopic rope hadronization model. The formation of ropes due to overlapping of strings followed by string shoving due to the creation of pressure gradient in the overlap region could generate the long range correlations in the near-side region of $\Delta \eta-\Delta \phi$ distribution for high multiplicity events in $p$ - $p$ collisions. This near-side ridge-like structure observed in the long range region is qualitatively similar to the ones measured in high multiplicity $p$ - $p$ collisions at LHC and thus can provide an alternate view of ridge formation when the formation of a thermalized medium is not imperative.

\section{ACKNOWLEDGMENTS}

The authors would like to thank Edward Sarkisyan Grinbaum and Miguel-Angel Sanchi for initiating the work and stimulating discussions throughout. The authors would like to thank the Department of Science and Technology (DST), Government of India for supporting the present work.
[1] V. Khachatryan et al. (CMS Collaboration), J. High Energy Phys. 09 (2010) 091.

[2] V. Khachatryan et al. (CMS Collaboration), Phys. Rev. Lett. 116, 172302 (2016).

[3] G. Aad et al. (ATLAS Collaboration), Phys. Rev. Lett. 116, 172301 (2016).

[4] J. Adams et al. (STAR Collaboration), Phys. Rev. Lett. 95, 152301 (2005).

[5] S. Chatrchyan et al. (CMS Collaboration), J. High Energy Phys. 07 (2011) 076.

[6] B. H. Alver, C. Gombeaud, M. Luzum, and J. Y. Ollitrault, Phys. Rev. C 82, 034913 (2010).

[7] B. Schenke, S. Jeon, and C. Gale, Phys. Rev. Lett. 106, 042301 (2011).

[8] H. Petersen, G. Y. Qin, S. A. Bass, and B. Muller, Phys. Rev. C 82, 041901(R) (2010).

[9] D. Teaney and L. Yan, Phys. Rev. C 83, 064904(R) (2011).

[10] K. Alpgrd et al. (UA5 Collaboration), Phys. Lett. B 123, 361 (1983).

[11] S. Chatrchyan et al. (CMS Collaboration), Phys. Lett. B 718, 795 (2013).

[12] K. Werner, I. Karpenko, and T. Pierog, Phys. Rev. Lett. 106, 122004 (2011).

[13] K. Dusling and R. Venugopalan, Phys. Rev. Lett. 108, 262001 (2012).

[14] A. Dumitru, K. Dusling, F. Gelis, J. J. Marian, T. Lappi, and R. Venugopalan, Phys. Lett. B 697, 21 (2011).

[15] M.-A. Sanchis-Lozano and E. Sarkisyan-Grinbaum, Phys. Lett. B 766, 170 (2017).

[16] M.-A. Sanchis-Lozano and E. Sarkisyan-Grinbaum, Phys. Rev. D 96, 074012 (2017).
[17] C. Andrés, A. Moscoco, and C. Pajares, Phys. Rev. C 90, 054902 (2014).

[18] M. A. Beaun, C. Pajares, and V. V. Vechernin, Eur. Phys. J. A 51, 44 (2015).

[19] T. Sjostrand, S. Ask, J. R. Christiansen, R. Corke, N. Desai, P. Ilten, S. Mrenna, S. Prestel, C. O. Rasmussen, and P. Z. Skands, Comput. Phys. Commun. 191, 159 (2015).

[20] C. Flensburg, G. Gustafson, and L. Lonnblad, J. High Energy Phys. 08 (2011) 103.

[21] C. Bierlich, G. Gustafson, and L. Lonnblad, MCnet-16-48, LUTP 16-64 (2016).

[22] R. Nayak, S. Pal, and S. Dash, Phys. Rev. D 100, 074023 (2019).

[23] C. Bierlich, Nucl. Phys. A 982, 499 (2019).

[24] C. Bierlich, EPJ Web Conf. 171, 14003 (2018).

[25] A. Goswami, R. Nayak, B. K. Nandi, and S. Dash, arXiv:1911.00559.

[26] C. Bierlich and J. R. Christiansen, Phys. Rev. D 92, 094010 (2015).

[27] J. R. Christiansen and P. Z. Skands, J. High Energy Phys. 08 (2015) 003.

[28] T. S. Biro, H. B. Nielson, and J. Knoll, Nucl. Phys. B 245, 449 (1984).

[29] C. Bierlich, G. Gustafson, L. Lonnblad, and A. Tarasov, J. High Energy Phys. 03 (2015) 148.

[30] P. Skands, S. Carrazza, and J. Rojo, Eur. Phys. J. C 74, 3024 (2014).

[31] N. N. Ajitanand, J. M. Alexander, P. Chung, W. G. Holzmann, M. Issah, Roy A. Lacey, A. Shevel, A. Taranenko, and P. Danielewicz, Phys. Rev. C 72, 011902(R) (2005). 